\title{
An Algorithm for Face Recognition based on Isolated Image Points with Neural Network
}

\author{
Hassan Jaleel Hassan, PhD \\ Computer Engineering Department, \\ University of Technology
}

\author{
Ali Kamal Taqi \\ BSc. \\ Computer Engineering Department, \\ University of Technology
}

\begin{abstract}
On the last years, face recognition has become a popular area of research in computer vision and one of the most successful applications of image analysis and understanding. Face recognition is of two types. One is pixel-based and the other is feature-based. Pixel-based techniques use principal component analysis (PCA) for face recognition, whereas feature-base techniques extract the facial features and use them to classify faces. Feature-based technique has been used in this work. Feature-based techniques extract the features of the face and use it for recognition. The recognition system should be robust enough to classify the face. Therefore, the training set should contain all the orientations of the face. In this work, the front view has only been taken in to consideration assuming that each person should stand in front of camera. The first step of the proposed algorithm is to resolve the image to Red, Green and Blue bands, then deal with each image as a gray scale one which is represented as a 2-D matrix. The second step is to detect isolated image points using simple method and alternative method. The third step is to extract features from each band. Finally, extracted features should be trained by neural network structure. Ten images have been tested by the proposed algorithm and the result of accuracy rate was $100 \%$.
\end{abstract}

\section{Keywords}

Face recognition, Feature extraction, Facial feature detection, Biometric identification, Recognition based on neural network

\section{INTRODUCTION}

In image processing, the inputs are images, and the outputs are also images. While in computer vision the inputs are images, but its outputs are attributes extracted from those images. This area includes tasks like image segmentation [1].

A face recognition system is a computer vision and it automatically identifies a human face from database images. The face recognition problem is challenging as needs to account for all possible appearance variation caused by change in illumination, facial features, occlusions, etc. [2].

In this paper, the proposed algorithm is based on image segmentation technique. Image segmentation is an important phase in image-based applications. Partitioning a digital image in to multiple regions and extracting a meaningful one is called image segmentation [3]

Image segmentation is one of image analysis methods used to subdivide an image into its regions or objects depending on the type of shapes and objects searched for in the image. Image segmentation is an essential first step in most automatic pictorial pattern recognition and scene analysis tasks.

Image segmentation algorithms are based on one of two basic properties of gray level values: discontinuity and similarity. In the first category, the approach is to partition an image based on abrupt discontinuity in gray level, such as edges in an image. In the second category, the approaches are based on partitioning an image into regions that are similar according to a set of predefined criteria [4]. In this paper we shall propose segmentation algorithm to detect discontinuity such as points.

One of developed approaches to pattern recognition has been the use of artificial neural network, which are now finding application in a wide variety of scientific disciplines. Neural networks are massively parallel distributed processing systems, which improve their performance through dynamical learning. The states of processing elements (neurons) can represent the elements of the adjustable model, which is compared with the system to be identified. To minimize the error between the system and the model, the learning rule is designed in such a way [5].

There are several related works proposed for face recognition that can be briefly demonstrated as follow: Rowely, H. A. et al. [6] present face detection system based on neural network. A neural network tests small windows of an image and make a decision if each window contains a face.

Phillips et al. [7] present overview of the face recognition grand challenge. This work describes the challenge problem, data corpus, and presents baseline performance and preliminary results on natural statistics of facial imager.

Ahonen, T. et al. [8] Present an efficient facial image representation depending on binary pattern features. The face image is partitioned into many regions from which pattern feature distributions are extracted and constructed as a feature vector to be used as a face classifier.

Naseem, I. et al. [9] introduce a method of face identification by considering the pattern recognition problem as a linear regression. They compensate a linear model of a probe image as a linear combination of class-specific galleries. The leastsquares method used to solve the inverse problem and the decision is ruled with the minimum reconstruction error.

In this work, isolated image points using simple method and alternative method are used to extract faces features. Finally, extracted features should be trained by neural network structure.

\section{DETECTION OF ISOLATED POINT $[3,4]$}

There are two methods of isolated point detection:

\subsection{Simple Method}

Point detection is concerned with detecting isolated image points in relation to its neighborhood which is an area of nearly constant gray level. We know that point detection 
should be based on the second derivative. This implies using the Laplacian:

$$
\nabla^{2} f(x, y)=\frac{d^{2} f}{d x^{2}}+\frac{d^{2} f}{d y^{2}}
$$

Where:

$$
\frac{d^{2} f(x, y)}{d x^{2}}=f(x+1, y)+f(x-1, y)-2 f(x, y)
$$

And

$\frac{d^{2} f(x, y)}{d y^{2}}=f(x, y+1)+f(x, y-1)-2 f(x, y)$

The laplacian is then

$$
\begin{array}{r}
\nabla^{2} \mathrm{f}(\mathrm{x}, \mathrm{y})=\mathrm{f}(\mathrm{x}+1, \mathrm{y})+\mathrm{f}(\mathrm{x}-1, \mathrm{y})+\mathrm{f}(\mathrm{x}, \mathrm{y}+1) \\
+\mathrm{f}(\mathrm{x}, \mathrm{y}-1)-4 \mathrm{f}(\mathrm{x}, \mathrm{y}) \ldots \ldots \ldots
\end{array}
$$

This expression can be implemented using the mask in figure (1) below:

\begin{tabular}{|c|c|c|}
\hline 1 & 1 & 1 \\
\hline 1 & -8 & 1 \\
\hline 1 & 1 & 1 \\
\hline
\end{tabular}

\section{Figure (1): Point detection Laplacian Mask}

The response of the mask at the center point of the region is:

$\mathrm{R}=\sum_{\mathrm{k}=1}^{9} \mathrm{w}_{\mathrm{k}} \mathrm{z}_{\mathrm{k}}$

Where $\mathrm{z}_{\mathrm{k}}$ is the intensity of the pixel whose spatial location corresponds to the location of the kth coefficient in the mask $\left(\mathrm{w}_{\mathrm{k}}\right)$. We say that a point has been detected at the location( $\mathrm{x}$, y) on which the mask at that point exceeds a specified threshold. Such points are labeled 1 in the output image and all other are labeled 0 , thus producing binary image. In other words, the output is obtained using the following expression:

$g=\left\{\begin{array}{lr}1 & \text { if }|R(x, y)| \geq T \\ 0 & \text { otherwise }\end{array}\right\}$

Where, $\mathrm{g}$ is the output image, $\mathrm{T}$ is a nonnegative threshold, and $\mathrm{R}$ is given by Eq. (5).

\subsection{Alternative Method}

An alternative approach to the simple method is to locate the points in a window of a given size where the difference between the max. and the min. value in the window exceeds a given threshold.

\section{FACE FEATURE EXTRACTION}

To extract object features, we need image segmentation and any necessary morphological filtering. This will provide us with clearly defined objects, which can then be labeled and processed independently. After all the objects in the image are labeled, we can treat each object as a binary image by assuming that the labeled object has a value of "1" and that everything else is "0". After we have labeled the objects, we have an image filled with object numbers. This image is used to extract the features of interest. The binary object features we will define include area, Euler number and standard deviation.

In order to provide general equation of area, a function $\mathrm{I}_{\mathrm{i}}(\mathrm{r}, \mathrm{c})$ is defined as [10]:
$I_{i}(r, c)\left\{\begin{array}{lr}1 & \text { if } I(r, c)=\text { ith object number } \\ 0 & \text { otherwise }\end{array}\right\}$

Where, $\mathrm{r}$ and $\mathrm{c}$ are the image matrix coordinates.

Now the area can be defined as:

$A_{i}=\sum_{r=0}^{N-1} \sum_{c=0}^{N-1} I_{i}(r, c)$

The Euler number of an image can be defined as the number of convexities minus the number of concavities, which are found by scanning the image for the patterns that follow.

Convexities

Concavities

$\left[\begin{array}{ll}0 & 0 \\ 0 & 1\end{array}\right]$

$\left[\begin{array}{ll}0 & 1 \\ 1 & 1\end{array}\right]$

The standard deviation of an image can be calculated using the following equation:

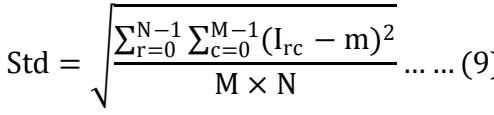

Std: the standard deviation.

$\mathrm{I}_{\mathrm{rc}}$ : Pixels of image matrix.

M: Mean of image matrix

M, N: Dimension of image matrix

\section{PROPOSED ALGORITHM}

The proposed algorithm consists of three phases: Building the reference set phase, training phase and recognition phase.

\subsection{Building the reference set}

This stage deals with the problem of constructing reference set. The procedures of generating face signature are given below;

1. Insert color face image into the algorithm.

2. Resolve the image to Red, Green and Blue bands.

3. Calculate standard deviation for each monochrome image.

4. Apply Simple method and Alternative method to determine the isolated points on each monochrome image.

5. Convert the resultant bands to black and white

6. Calculate Area, Euler number for resultant bands.

7. Save the calculated values for three monochrome images in one vector which represents the main feature vector (face signature).

8. Repeat all above steps for all images related with proposed algorithm. 


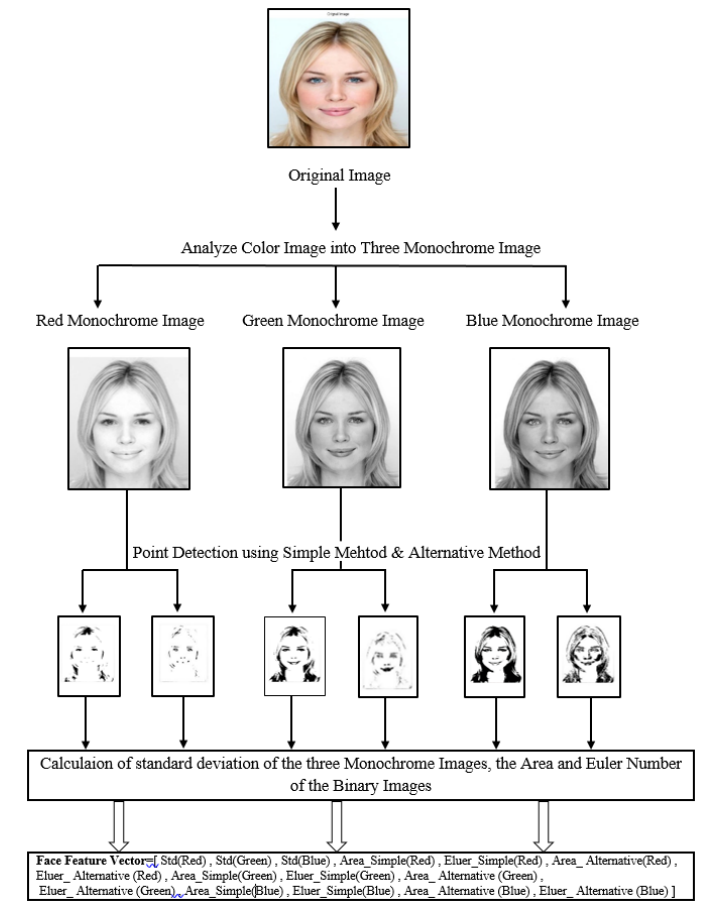

Fig 2: shows the steps of building the feature vector for the face color image taken by a digital camera.

\subsection{Training phase}

The neural network (NN) construction for face recognition is a feed forward multi-layer net with an input layer, two hidden layer and output layer. Back propagation is used for training algorithm. After many tests the proper choice to the neural network structure was 18 neurons in the input layer which represent the number of feature vector used in classification, 10 neurons in the first hidden layer, 20 neurons in the second hidden layer, and 10 neurons in the output layer which represent the number of images in the application algorithm working with. The network continues training with the input patterns until $\left(\mathrm{E}=10^{-7}\right)$ reaches, where $\mathrm{E}$ represent the mean square error values between the values of desired and target vectors, then save the file after training stops, which contain all the training weights variables to use it in testing phase. Figure (3) shows a flowchart for the NN training procedure and training steps to reach the desired goal is shown in figure (4)

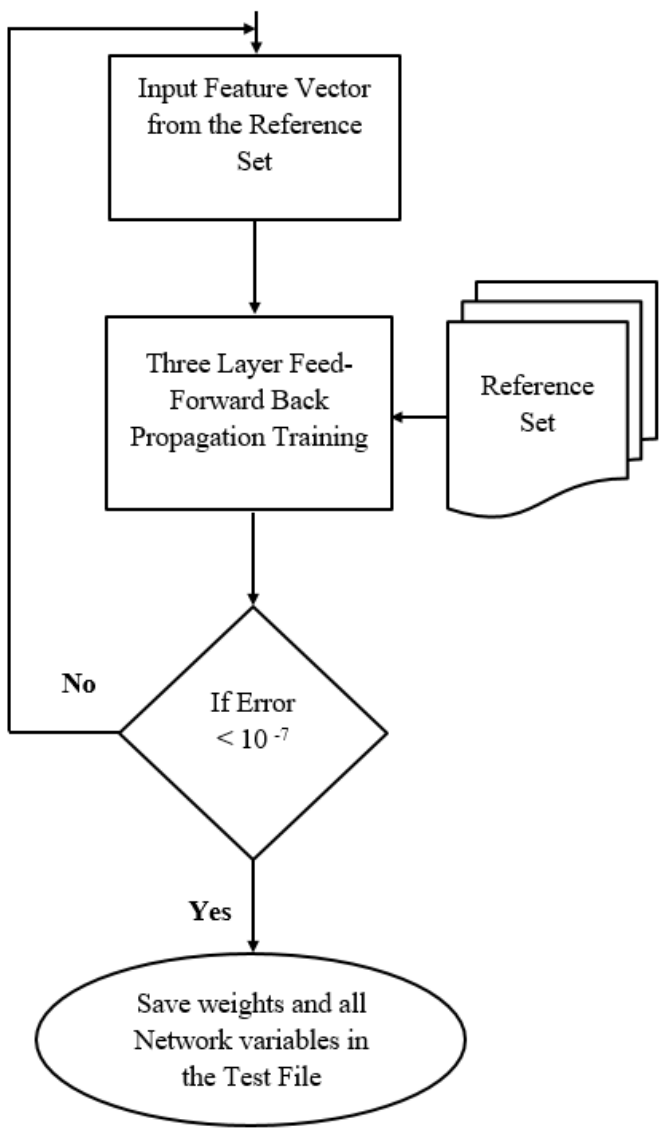

Fig 3: Flowchart for the NN training procedure

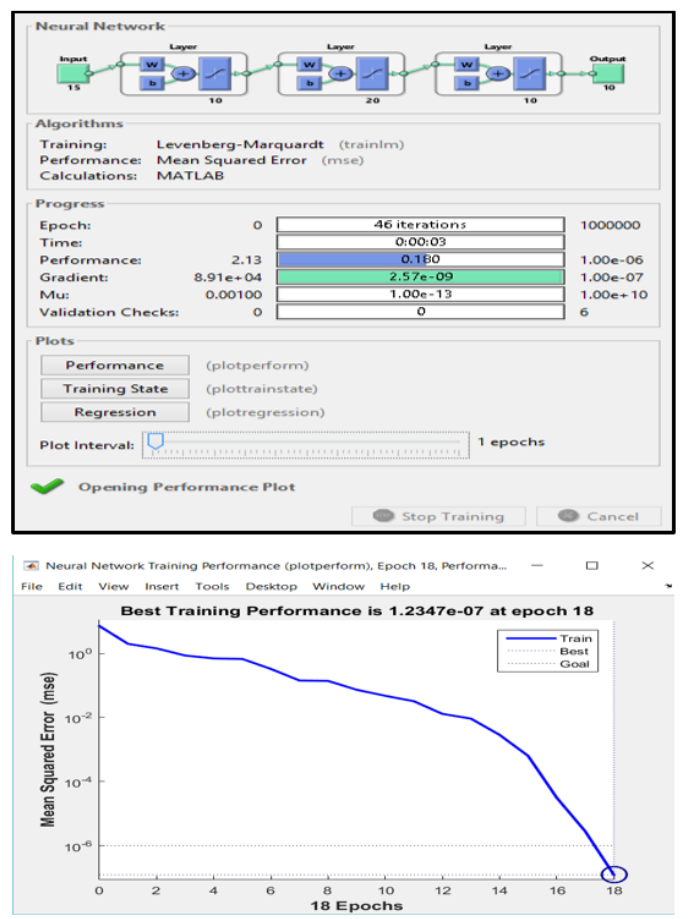

Fig 4: Neural Network Training steps to reach the desired goal

\subsection{Recognition phase}

Figure (5) shows a block diagram for recognition phase using NN. neurons input layer receive the direct input feature values from the pattern to be classified. The output layer has one 
perceptron element per data class and should compute a high output signal at the appropriate element representing the correct class for this pattern. In practically this will happen if network weights values closed to the connections have been learnt.

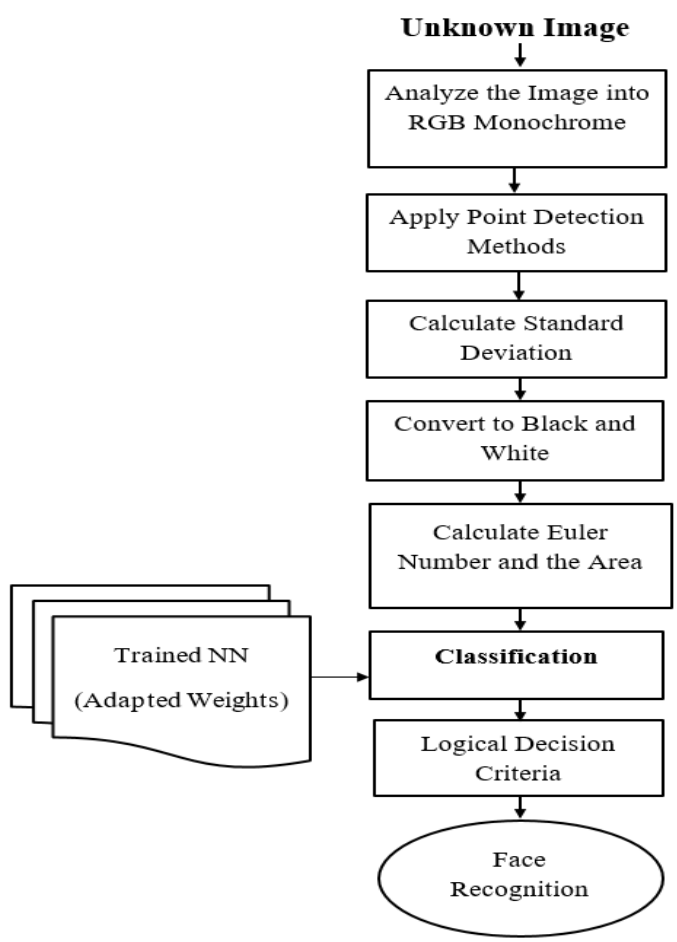

In this work, ten face images for different persons are used in building the reference set. These images have been extracted from the location (http://www.goldennumber.net/wpcontent/uploads/2013/08/fl orence-colgate-england-most-beautiful-face.jpg). Table (1) shows the results of all ten feature vectors which contain the calculated value of Area, Euler number, and standard deviation for all ten color images represented by their monochrome bands.

Fig 5: Block Diagram for Recognition training procedure

\section{RESULTS}

Table 1. The Results of All Ten Feature Vectors

\begin{tabular}{|c|c|c|c|c|c|c|c|c|c|c|c|c|c|c|c|}
\hline \multicolumn{16}{|c|}{$\boxplus$ 10x15 double } \\
\hline & 1 & 2 & 3 & 4 & 5 & 6 & 7 & 8 & 9 & 10 & 11 & 12 & 13 & 14 & 15 \\
\hline 1 & 81.2502 & 92.8818 & 96.7328 & 88980 & -9 & 85592 & -13 & 84762 & -15 & 110652 & -68 & 108252 & -94 & 105860 & -44 \\
\hline 2 & 85.9207 & 90.7336 & 93.8332 & 94150 & -49 & 89900 & -63 & 87046 & -33 & 109150 & -43 & 105984 & -75 & 99206 & 11 \\
\hline 3 & 84.6052 & 90.6241 & 94.0297 & 92478 & -98 & 81516 & -45 & 76526 & -16 & 109170 & -29 & 103056 & -207 & 90760 & -2 \\
\hline 4 & 68.6150 & 81.1803 & 90.0252 & 99054 & -22 & 91700 & -56 & 79778 & 18 & 109044 & -53 & 107672 & -170 & 89334 & -5 \\
\hline 5 & 31.6451 & 64.4673 & 81.4581 & 112970 & -15 & 103654 & -155 & 87456 & -83 & 112940 & -36 & 88356 & -27 & 66854 & 89 \\
\hline 8 & 52.6536 & 75.1420 & 83.6476 & 106930 & -13 & 96614 & -97 & 91416 & -76 & 110224 & -72 & 93926 & -13 & 82526 & -8 \\
\hline 9 & 84.0184 & 89.2142 & 91.8178 & 96578 & -24 & 89242 & -59 & 86500 & -6 & 110154 & -31 & 107052 & -174 & 102430 & -38 \\
\hline 10 & 59.2706 & 60.7562 & 70.1890 & 106276 & -35 & 104772 & -34 & 102222 & -37 & 111502 & -66 & 107648 & -200 & 85836 & 28 \\
\hline
\end{tabular}

\section{CONCLISIONS}

The objective of this paper is to develop an algorithm for face recognition with neural network based on face features. Toward this goal, an algorithm depending on extraction gray and binary object features has been proposed. The gray features included standard deviation while, binary object features included Area, and Euler Number. Area tells us something about when the object is. Euler Number tells us something about shape of the object while standard deviation tells us about how the gray levels distribution.

The conclusion points can be briefly states as follow.

1. The proposed algorithm reflected the effectiveness of using simple method filter to find the implicit information in the face image, so as alternative.

2. Because face images exist in different colors, this feature gave us a wide range to extract implicit information, and that increased the robustness of the proposed algorithm

3. The neural networks often give high classification rate than parametric methods (minimum distance methods)

4. Neural network has the ability of self-learning which achieve an accurate result of recognition of face images of $100 \%$.

\section{REFERENCES}

[1] Riedmiller, M., Braun, H., " A direct adaptive method for faster backpropagation learning: the RPROP algorithm", proc. Of the IEEE Int. conf. on neural networks, 1993. 
[2] P.Latha, Dr. L. Ganeasan and Dr. S. Annadurai.,"Face Recognition using neural network", Intrnational Journal of signal processing. Vol. 3, Issue 5, pp. 153.

[3] S. Sridhar,"Digital Image Processing", Oxford University Press, 2011.

[4] Gonzalez, R. C., Woods, R. E.,"Digital Image Processing", Third Edition, Pearson Prentice Hall, 2009.

[5] Chitra, S. P., " Use neural network for problem solving”, Chemical Engineering Progress, pp. 44-52, April 1993.

[6] Rowley, H.A., Baluja, S., Kanade, T., “ Neural network-based face detection", Pattern Analysis and Machine Intelligence, IEEE Transactions. Vol. 20 , Issue: 1 PP. 23-38. 1998.
[7] Phillips, P.J. ; Flynn, P.J. ; Scruggs, T. ; Bowyer, K.W. "Overview of the face recognition grand challenge", Computer Vision and Pattern Recognition. IEEE Computer Society Conference. Vol.1. 2005.

[8] Ahonen, T. Hadid, A. ; Pietikainen, M., "Face Description with Local Binary Patterns: Application to Face Recognition", Pattern Analysis and Machine Intelligence, IEEE Transactions. Vol. 28, Issue: 12, PP. 2037-2041. 2006.

[9] Naseem, I. ; Togneri, R. ; Bennamoun, M. , "Linear Regression for Face Recognition", Pattern Analysis and Machine Intelligence, IEEE Transactions. Vol. 32, No.11, PP. 2106-2112. 2010.

[10] Gonzalez, R. C., Woods, R. E.,"Digital Image Processing", Adison-Wesely Publishing Company 1992. 